\title{
Electrical and Structural Properties of ZnO Synthesized via Infiltration of Lithographically-Defined Polymer Templates
}

\author{
Chang-Yong Nam, ${ }^{\text {a) }}$ Aaron Stein, Kim Kisslinger, and Charles T. Black \\ Center for Functional Nanomaterials, Brookhaven National Laboratory \\ Upton, New York 11973, USA
}

We investigate electrical and structural properties of infiltration-synthesized $\mathrm{ZnO}$. In-plane $\mathrm{ZnO}$ nanowire arrays with prescribed positional registrations are generated by infiltrating diethlyzinc and water vapor into lithographically defined SU-8 polymer templates and removing organic matrix by oxygen plasma ashing. Transmission electron microscopy reveals homogeneously amorphous as-infiltrated polymer templates transform into highly nanoscrystalline $\mathrm{ZnO}$ upon removal of organic matrix. Field-effect transistor device measurements show that the synthesized $\mathrm{ZnO}$ after thermal annealing displays a typical $\mathrm{n}$-type behavior, $\sim 10^{19} \mathrm{~cm}^{-3}$ carrier density, and $\sim 0.1 \mathrm{~cm}^{2} \mathrm{~V}^{-1} \mathrm{~s}^{-1}$ electron mobility, reflecting highly nanocrystaline internal structure. The results demonstrate the potential application of infiltration synthesis in fabricating metal oxide electronic devices.

\footnotetext{
${ }^{a)}$ Author to whom correspondence should be addressed. Electronic mail: cynam@bnl.gov
} 
Vapor-phase infiltration of patterned polymeric templates is an emerging material synthesis strategy derived from atomic layer deposition during which sequentially introduced gaseous material precursors perfuse and react within the polymer, generating localized organic-inorganic hybrids that may be subsequently converted to all inorganic. Different versions of this method, including multi-pulse infiltration (MPI), ${ }^{1-3}$ sequential vapor infiltration (SVI), ${ }^{4,5}$ and sequential infiltration synthesis (SIS),${ }^{67}$ have proven useful for enhancing the physical properties of polymers, for example by increasing their mechanical strength ${ }^{1-3,8,9}$ and by improving their etch resistance. ${ }^{10-13}$ Further, the polymer material may be removed completely, generating patterned metal oxide structures having the dimensions of the initial template. ${ }^{4,6,7,14}$ Examples include mesoporous metal oxide microfibers with controlled nanoscale porosity synthesized by applying SVI to polyester (PE) microfibers, ${ }^{4}$ and metal oxide nanostructures created using SIS of selfassembled poly(styrene- $b$-methylmethacrylate) (PS- $b$-PMMA) and poly(styrene- $b$-poly-2-vinyl pyridine) (PS- $b$-P2VP) block copolymer thin films. ${ }^{6,714,15}$ In this work, we investigate the electrical and structural properties of nanostructured $\mathrm{ZnO}$ synthesized by infiltration of lithographically defined polymeric templates, in order to understand the electronic quality of materials produced by this approach and assess new opportunities for applying this type of synthesis in electronic device fabrication.

We generate $\mathrm{ZnO}$ nanowire arrays by sequential vapor-phase infiltration of diethylzinc (DEZ) and water vapor into lithographically patterned SU-8 polymer templates. We recently reported the nanopatterning of $\mathrm{TiO}_{\mathrm{x}}$ nanowire arrays and high aspect ratio $(\sim 16) \mathrm{AlO}_{\mathrm{x}}$ nanostructures with sub-40 $\mathrm{nm}$ linewidth by using a similar approach. ${ }^{16}$ In this study, we monitor the morphological evolution of the SU-8 template throughout the synthesis to quantify the extent of $\mathrm{ZnO}$ infiltration, characterize the nanocrystalline $\mathrm{ZnO}$ internal structure by high-resolution transmission electron 
microscopy (TEM), and finally understand the material's electrical properties through fieldeffect transistor (FET) measurements. Notably, we find that suitably post-processed infiltrationsynthesized $\mathrm{ZnO}$ is an $\mathrm{n}$-type semiconductor with carrier concentration of $\sim 10^{19} \mathrm{~cm}^{3}$ and electron mobility of $\sim 0.1 \mathrm{~cm}^{2} \mathrm{~V}^{-1} \mathrm{~s}^{-1}$, which is in the range of mobility values of other deposited thin film semiconductors such as organics ${ }^{17}$ nanocrystals, ${ }^{18}$ and amorphous silicon. ${ }^{19}$

We generated SU-8 wire templates with $5 \mu \mathrm{m}$ nominal length and cross-sectional dimensions of $100 \mathrm{~nm}$ width and $130 \mathrm{~nm}$ height, using an electron beam (e-beam) lithography (Figures 1(a) and $1(\mathrm{~b})) .{ }^{20} \mathrm{~A}$ commercial atomic layer deposition (ALD) system (at $95^{\circ} \mathrm{C}$ ) was then used to sequentially expose the SU-8 templates to DEZ and water vapor (repeating this cycle four times), with each exposure lasting $300 \mathrm{~s}$ (at a vapor pressure <10 Torr) (Figure 1(c)). We expect that Lewis basic C-O functional groups within the SU-8 (Figure 1(e)) facilitate the retention of infused Lewis-acidic DEZ (Figure (f)) via a binding reaction. Finally, oxygen plasma ashing (20 W, 100 mTorr, $5 \mathrm{~min}$ ) converts the infiltrated SU-8 templates into $\mathrm{ZnO}$ nanostructures (Figure (d)).

Infiltration synthesis of lithographically patterned $\mathrm{SU}-8$ templates generates $\mathrm{ZnO}$ structures with controlled geometries and spatial positions (Figures 1(g) and 1(h)); we fabricated arrays of in-plane $\mathrm{ZnO}$ nanowires (length $5 \mu \mathrm{m}$ and width $\sim 50 \mathrm{~nm}$ ) with precisely prescribed positional registrations in order to study the electronic properties using a FET geometry. Aside from being an excellent tool for material evaluation, FETs form the foundation of computational electronics and many types of sensors. The synthesized $\mathrm{ZnO}$ wires are significantly smaller in size from the parent SU-8 template as a result of the infiltration and template removal. This reflects the finite quantity of $\mathrm{ZnO}$ loaded during the infiltration process. A nanowire geometry is well suited to studies of the volume changes through cross-sectional imaging. Compared to the starting SU-8 
template dimensions of $100 \pm 7 \mathrm{~nm}$ (width) and $133 \pm 4 \mathrm{~nm}$ (height) (Figure 2(a) and 2(d)), after four DEZ/water vapor infiltration cycles the SU-8 templates swell in both width and height by $\sim 7 \%$ (to $106 \pm 7 \mathrm{~nm}$ and $144 \pm 5 \mathrm{~nm}$, respectively) (Figures 2(b) and 2(d)), reflecting metal oxide loading within the polymer matrix. The total volume increase of the nanostructure is $\sim 15 \%$. However, after polymer template removal, the final structure has a $~ 61 \%$ smaller volume, compared with the initial polymer, with lateral dimensions decreasing to (width) $54 \pm 8 \mathrm{~nm}$ and (height) $95 \pm 8 \mathrm{~nm}$ (Figures 2(c) and 2(d)). These extents of dimensional evolution are similar to what was observed for the infiltration synthesis of $\mathrm{AlO}_{\mathrm{x}}$ in $\mathrm{SU}-8,{ }^{16}$ thus suggesting that the interaction and loading of DEZ in SU-8 are comparable to those of trimethylaluminium $\left(\mathrm{AlO}_{\mathrm{x}}\right.$ precursor) in SU-8.

Bright-field TEM images of the SU-8 wire template cross-section show a homogenously amorphous material after four sequential DEZ/water vapor exposures (Figure 3(a)). We prepared TEM samples by Ga-ion-beam milling in-plane $\mathrm{ZnO}$ nanowires protected by a $\mathrm{SiO}_{\mathrm{x}}$ cap made from gaseous tetraethyl orthosilicate (TEOS). ${ }^{20}$ Chemical analysis of the organic-inorganic composite by energy dispersive X-ray spectroscopy (EDXS) shows the SU-8 template is uniformly infiltrated with $\mathrm{ZnO}$, evidenced primarily by the $\mathrm{Zn}_{\alpha 1}$ signal's constant elevation across the wire cross-section (Figure $3(\mathrm{~b})$ ). The oxygen $\mathrm{K}_{\alpha 1}$ counts within in the ZnO-infiltrated template are lower than those in the $\mathrm{SiO}_{\mathrm{x}}$ capping layer, consistent with the lower nominal oxygen atomic concentration of $\mathrm{ZnO}(50 \%)$ than that of $\mathrm{SiO}_{2}(\sim 67 \%)$. The non-uniform oxygen signal profile near edges most likely results from oxygen spill-over from the $\mathrm{SiO}_{\mathrm{x}}$ capping region. Efficient diffusion and sorption of water within micrometer-scale SU-8 structures has been documented in previous studies, ${ }^{21}$ and so we expect that insufficient DEZ retention within the 
SU-8 template is a primary factor determining the amount of $\mathrm{ZnO}$ loading by infiltration synthesis.

From the measured volume of the final inorganic structure (Figures 2(c) and 2(d)) and the volume decrease compared to the as-infiltrated composite ( 66\%) (Figures 2(b) and 2(d)), we deduce that $\mathrm{ZnO}$ occupies $34 \%$ of the total volume of the composite. We anticipate that the densified organic-inorganic hybrid will have increased mechanical strength compared to SU-8, similar to the previous report for metal-oxide-impregnated spider silks. ${ }^{1}$ Because the DEZ precursor is expected to selectively bind to the C-O groups in the SU-8, each exposure should attach at most 16 zinc atoms to each SU-8 monomer in principle. From the SU-8 bulk density $\left(\sim 1.2 \mathrm{~g} / \mathrm{cm}^{3}\right)$ and the monomer molar mass $(1,397 \mathrm{~g} / \mathrm{mol})$, we can estimate the number of direct DEZ attachment sites as $\sim 8 / \mathrm{nm}^{3}$ within the SU-8. Interestingly, we find approximately twice more DEZ molecules infiltrated compared with the total number of attachment sites, considering the final dimension of synthesized $\mathrm{ZnO}$ and its nominal density $\left(\sim 5.6 \mathrm{~g} / \mathrm{cm}^{3}\right) \cdot{ }^{20} \mathrm{We}$ speculate that the DEZ sorption in SU-8 may not be limited by the number of binding sites but rather by the available molecular-scale space, similar to the water sorption within SU-8. ${ }^{21}$

We probe the electrical properties of the infiltration-synthesized $\mathrm{ZnO}$ material by fabricating and measuring FET arrays having $1 \mu \mathrm{m}$ long $\mathrm{ZnO}$ wires (with cross-sectional areas of $\sim 34 \mathrm{~nm} \times$ $34 \mathrm{~nm})$ as the semiconducting channel. For these studies, we isolated the nanowires from the conducting silicon substrate with a $300 \mathrm{~nm} \mathrm{SiO}{ }_{2}$ layer and used the substrate as a gate to modulate carrier concentration within the material. Source/drain contacts (Ti/ $\mathrm{Au}, 10 \mathrm{~nm} / 30 \mathrm{~nm}$ ) to the $\mathrm{ZnO}$ channel were defined by e-beam lithography and thermal evaporation, based on the prescribed nanowire registration (Figure 4(a)). Both as-fabricated $\mathrm{ZnO}$ nanowires and those annealed in oxygen $\left(500{ }^{\circ} \mathrm{C}, 10 \mathrm{~min}\right.$, by a rapid thermal processor) were highly resistive (> 100 
$\mathrm{G} \Omega$ ) for all applied gate voltages, $V_{G}$. The infiltration-synthesized $\mathrm{ZnO}$ became semiconducting only after annealing in forming gas $\left(4 \% \mathrm{H}_{2}\right.$ with Ar balance, $\left.500{ }^{\circ} \mathrm{C}, 10 \mathrm{~min}\right)$. Interstitial hydrogens or oxygen vacancies are known to be n-type dopants in $\mathrm{ZnO}^{22}$ and we expect that the forming gas annealing activates as-synthesized $\mathrm{ZnO}$ nanowires by increasing the carrier concentration.

$\mathrm{ZnO}$ single nanowire FETs display n-channel electrical transport and drive currents of $\sim 120$ $\mathrm{nA}$ at source/drain voltage $\left(V_{D S}\right)$ of $2.5 \mathrm{~V}$ and gate voltage $\left(V_{G}\right)$ of $+60 \mathrm{~V}$ (Figure $4(\mathrm{~b})$ ). This corresponds to a current density of $\sim 10^{7} \mathrm{~mA} / \mathrm{cm}^{2}$ through the nanowire cross-section. The device transfer characteristics $\left(I_{D S}-V_{G}\right.$, Figure 4(c)) show a minimum channel conductance at $V_{G}$ $<-60 \mathrm{~V}$, from which we can estimate a carrier concentration $\left(N_{e}\right)$ of at least $2.5 \times 10^{19} \mathrm{~cm}^{-3}$ using the gate channel geometric capacitance (and assuming a cylindrical wire geometry and full channel depletion at $-60 \mathrm{~V}):^{23}$

$$
N_{e} \sim 2 \varepsilon_{o} \varepsilon_{r}\left|V_{T H}\right| /\left(q r^{2} \ln (2 t / r)\right)
$$

where $V_{T H}$ is the device threshold voltage $(-60 \mathrm{~V}), \varepsilon_{o}$ is the vacuum permittivity, $\varepsilon_{r}$ is the relative permittivity of $\mathrm{SiO}_{2}(\sim 3.9), q$ is the elementary charge, $r$ is the nanowire radius (assuming a circular cross-section), and $t$ is the gate oxide thickness $(300 \mathrm{~nm})$. The high carrier concentration likely limits the FET current on-off ratio, which we measure as a $~ 10 \times$ swing in zero-bias conductance $(G)$ as the gate voltage $\left(V_{G}\right)$ increases from $-60 \mathrm{~V}$ to $+60 \mathrm{~V}$ (Figure $4(\mathrm{~b})$ inset).

The nanowire FET transconductance $\left(d I_{D S} / d V_{G}\right)$ increases in proportion to $V_{D S}$ (Figure $4 \mathrm{c}$, inset), consistent with the expression:

$$
\left(d I_{D S} / d V_{G}\right)=\mu V_{D S}\left(2 \pi \varepsilon_{o} \varepsilon_{r} /(L \ln (2 t / r))\right.
$$

with $L$ the channel length and $\mu$ the charge carrier mobility. ${ }^{23}$ From the slope of $\left(d I_{D S} / d V_{G}\right)$ versus $V_{D S}$, we deduce an electron mobility within the synthesized $\mathrm{ZnO}$ of $\mu \sim 0.07 \mathrm{~cm}^{2} \mathrm{~V}^{-1} \mathrm{~s}^{-1}$, a 
value roughly $100-1000 \times$ lower than that reported for single crystal $\mathrm{ZnO}$ nanowires ${ }^{24}$ and consistent with the material's highly nanocrystalline internal structure (described below), where high density of grain boundaries can act as charge traps.

Cross-sectional TEM of the $\mathrm{ZnO}$ nanowire after polymer template removal reveals that the infiltration synthesis produces a highly nanocrystalline material. Low-magnification, bright-field TEM micrographs (Figure 3(c)) show a near rectangular nanowire cross-section, consistent with SEM images (Figure 2). Variation in the atomic number $(\mathrm{Z})$ contrast across the $\mathrm{ZnO}$ wire crosssections indicate low density areas within the nanostructure. The EDXS line scan of the nanowire cross-section consistently confirms the existence of $\mathrm{Zn}$ and oxygen (Figure 3(d)). High-resolution images show the wires are composed of nanocrystalline $\mathrm{ZnO}$ having grain sizes smaller than $\sim 5 \mathrm{~nm}$, with no amorphous regions visible within the field of view (Figure 3(e)). Fast Fourier transforms (FFT) of these micrographs show diffraction rings, consistent with random crystalline grain orientations (Figure 3(e) inset). Selected area electron diffraction (SAED) from the center of the wire cross-section resolves two pseudo diffraction rings (Figure 3(f)), which are consistent with a cubic $\mathrm{ZnO}$ crystal phase that is uncommon, but has been previously observed in a nanocrystalline $\mathrm{ZnO}$ thin film prepared by pyrolysis. ${ }^{25} \mathrm{We}$ expect that the brief post-synthesis carrier activation annealing applied at $500{ }^{\circ} \mathrm{C}$ for $10 \mathrm{~min}$ by rapid thermal processing should not render the $\mathrm{ZnO}$ internal structure significantly different from the asinfiltration-synthesized one, given the high melting point of $\mathrm{ZnO}\left(1,975^{\circ} \mathrm{C}\right)$. Further study is under progress currently to quantify the impact of different annealing conditions on the structural and electrical characteristics of infiltration-synthesized $\mathrm{ZnO}$, which eventually should help us identify optimal thermal processing conditions for specific applications (e.g., larger grain size for 
high electron mobility; high grain boundary density for efficient gas sensing; low processing temperature for applications in flexible substrates).

Infiltration synthesis provides a method of directly patterning $\mathrm{ZnO}$ material from lithographically defined polymer templates. We have investigated the electrical and structural properties of in-plane $\mathrm{ZnO}$ nanowire arrays synthesized by this approach, using a FET geometry to measure charge carrier concentration and mobility values. Electron microscopy measurements revealed the dimensional evolution of the polymer template during the synthesis process and upon organic template removal. Despite the relatively modest electrical performance of infiltration-synthesized $\mathrm{ZnO}$ in the current study, the demonstrated patterning capability of welldefined electrically active nanostructure arrays with precise positional registration provides a potential new route to high-throughput nano- or micropatterned metal oxide electronic device fabrication. For instance, mature optical lithography can be utilized to generate starting polymeric templates in a wafer scale, and they all can be converted into patterned electrically active metal oxide using a conventional atomic layer deposition system. We envision these studies of the electrical performance of infiltration-synthesized $\mathrm{ZnO}$ as a starting point, and that further improvements may be possible by improving the understanding on the chemical interaction between precursors and SU-8 during the infiltration synthesis and by controlling resulting material crystallinity and carrier concentration through an optimized post-synthesis thermal treatment.

This research was carried out at the Center for Functional Nanomaterials, Brookhaven National Laboratory (BNL), which is supported by the U.S. Department of Energy, Office of Basic Energy Sciences, under Contract No. DE-SC0012704. 
S. M. Lee, E. Pippel, U. Gosele, C. Dresbach, Y. Qin, C. V. Chandran, T. Brauniger, G. Hause and M. Knez, Science 324, 488 (2009).

S. M. Lee, V. Ischenko, E. Pippel, A. Masic, O. Moutanabbir, P. Fratzl and M. Knez, Adv. Funct. Mater. 21, 3047 (2011).

S. M. Lee, E. Pippel, O. Moutanabbir, I. Gunkel, T. Thurn-Albrecht and M. Knez, ACS Appl. Mater. Inter. 2, 2436 (2010).

B. Gong, Q. Peng, J. S. Jur, C. K. Devine, K. Lee and G. N. Parsons, Chem. Mater. 23, 3476 (2011).

H. I. Akyildiz, R. P. Padbury, G. N. Parsons and J. S. Jur, Langmuir 28, 15697 (2012).

Q. Peng, Y. C. Tseng, S. B. Darling and J. W. Elam, Adv. Mater. 22, 5129 (2010).

Q. Peng, Y. C. Tseng, S. B. Darling and J. W. Elam, ACS Nano 5, 4600 (2011).

S. M. Lee, E. Pippel and M. Knez, ChemPhysChem 12, 791 (2011).

S. M. Lee, E. Pippel, O. Moutanabbir, J. H. Kim, H. J. Lee and M. Knez, ACS Appl. Mater. Inter. 6, 16827 (2014).

Y. C. Tseng, Q. Peng, L. E. Ocola, D. A. Czaplewski, J. W. Elam and S. B. Darling, J. Vac. Sci. Technol. B 29, 06FG01 (2011).

Y. C. Tseng, Q. Peng, L. E. Ocola, D. A. Czaplewski, J. W. Elam and S. B. Darling, J. Mater. Chem. 21, 11722 (2011).

Y. C. Tseng, A. U. Mane, J. W. Elam and S. B. Darling, Adv. Mater. 24, 2608 (2012).

Y. C. Tseng, Q. Peng, L. E. Ocola, J. W. Elam and S. B. Darling, J. Phys. Chem. C. 115, 17725 (2011).

J. Kamcev, D. S. Germack, D. Nykypanchuk, R. B. Grubbs, C. Y. Nam and C. T. Black, ACS Nano 7, 339 (2013).

Pawel W. Majewski, Atikur Rahman, Charles T. Black and Kevin G. Yager, Nat.

Commun. 6, 7448 (2015).

C. Y. Nam, A. Stein and K. Kisslinger, J. Vac. Sci. Technol. B 33, 06F201 (2015).

J. Zaumseil and H. Sirringhaus, Chem. Rev. 107, 1296 (2007).

D. Vanmaekelbergh and P. Liljeroth, Chem. Soc. Rev. 34, 299 (2005).

K. Nomura, H. Ohta, A. Takagi, T. Kamiya, M. Hirano and H. Hosono, Nature 432, 488 (2004).

See supplemental material at [URL will be inserted by AIP] for experimental details.

K. Wouters and R. Puers, J. Micromech. Microeng. 20, 095013 (2010).

A. Janotti and C. G. Van de Walle, Rep. Prog. Phys. 72, 126501 (2009).

M. Radosavljevic, J. Appenzeller, P. Avouris and J. Knoch, Appl. Phys. Lett. 84, 3693 (2004).

Z. Y. Fan, D. W. Wang, P. C. Chang, W. Y. Tseng and J. G. Lu, Appl. Phys. Lett. 85, 5923 (2004).

M. Parhizkar, M. Kumar, P. K. Nayak, S. Singh, S. S. Talwar, S. S. Major and R. S.

Srinivasa, Colloid. Surface. A 257-258, 445 (2005). 

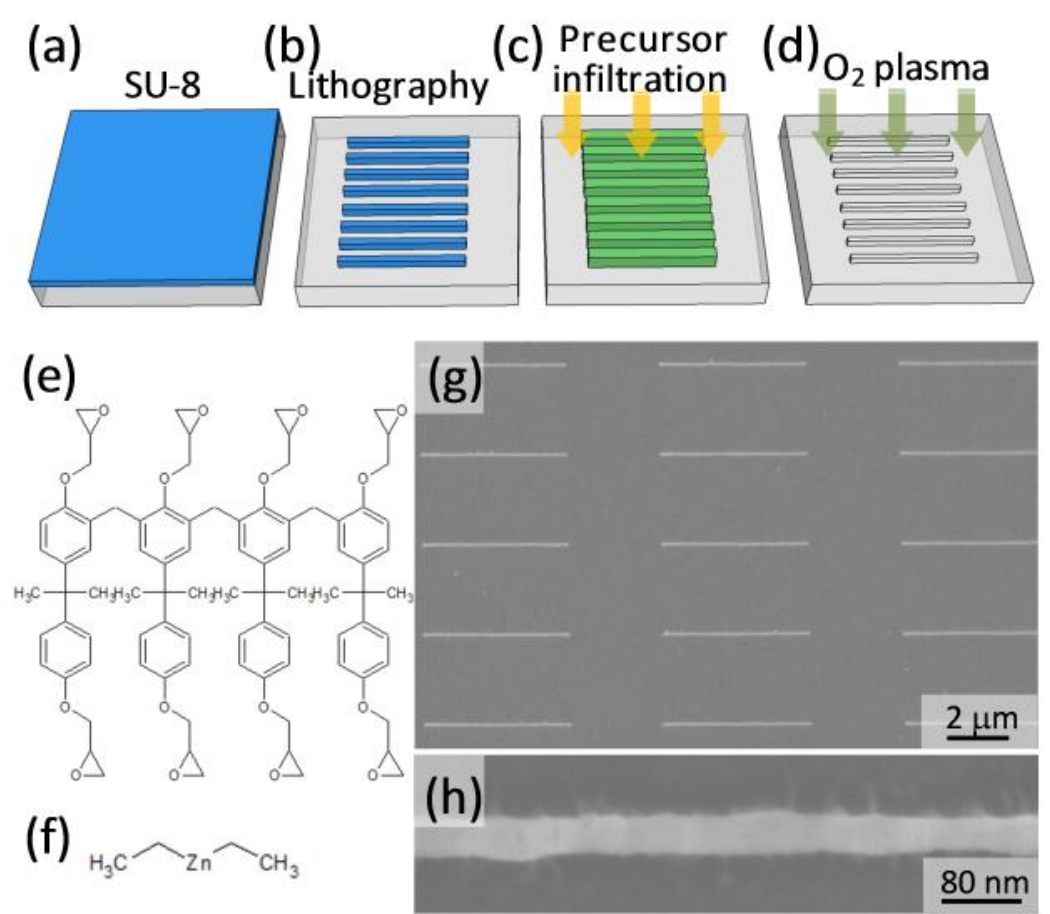

FIG. 1. Schematic description of the $\mathrm{ZnO}$ patterning process via the infiltration synthesis in lithographically defined topographical polymer templates: (a) SU-8 polymer film (blue) coating on a substrate (grey); (b) generation of patterned, topographical SU-8 templates by lithography; (c) infiltration synthesis of $\mathrm{ZnO}$ (yellow arrows) within the SU-8 templates; (d) final $\mathrm{ZnO}$ nanostructure formation by oxygen plasma ashing of the polymer templates. Nominal chemical structures of (e) SU-8 monomer and (f) DEZ molecule. (g) Top-down SEM micrograph of generated in-plane $\mathrm{ZnO}$ nanowire arrays and (h) high-magnification view of a section of $\mathrm{ZnO}$ nanowire. 

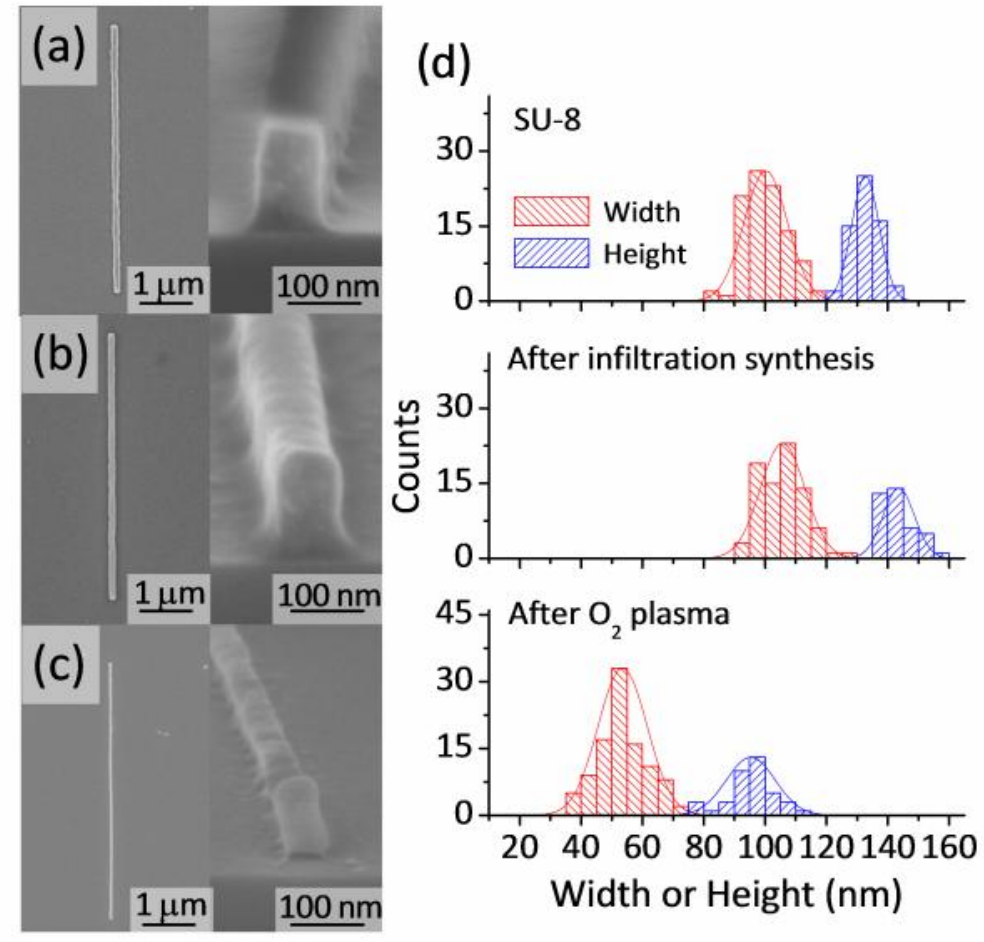

FIG. 2. Representative SEM micrographs (top (left) and 70-degree cross-sectional (right) views): (a) Initial SU-8 nanowire templates; (b) SU-8 templates after 4 cycles of $\mathrm{ZnO}$ infiltration synthesis; and (c) the final $\mathrm{ZnO}$ nanowire after oxygen plasma removal of the polymer matrix. (d) Histograms of size (width and height) distribution during each stage of infiltration synthesis. 

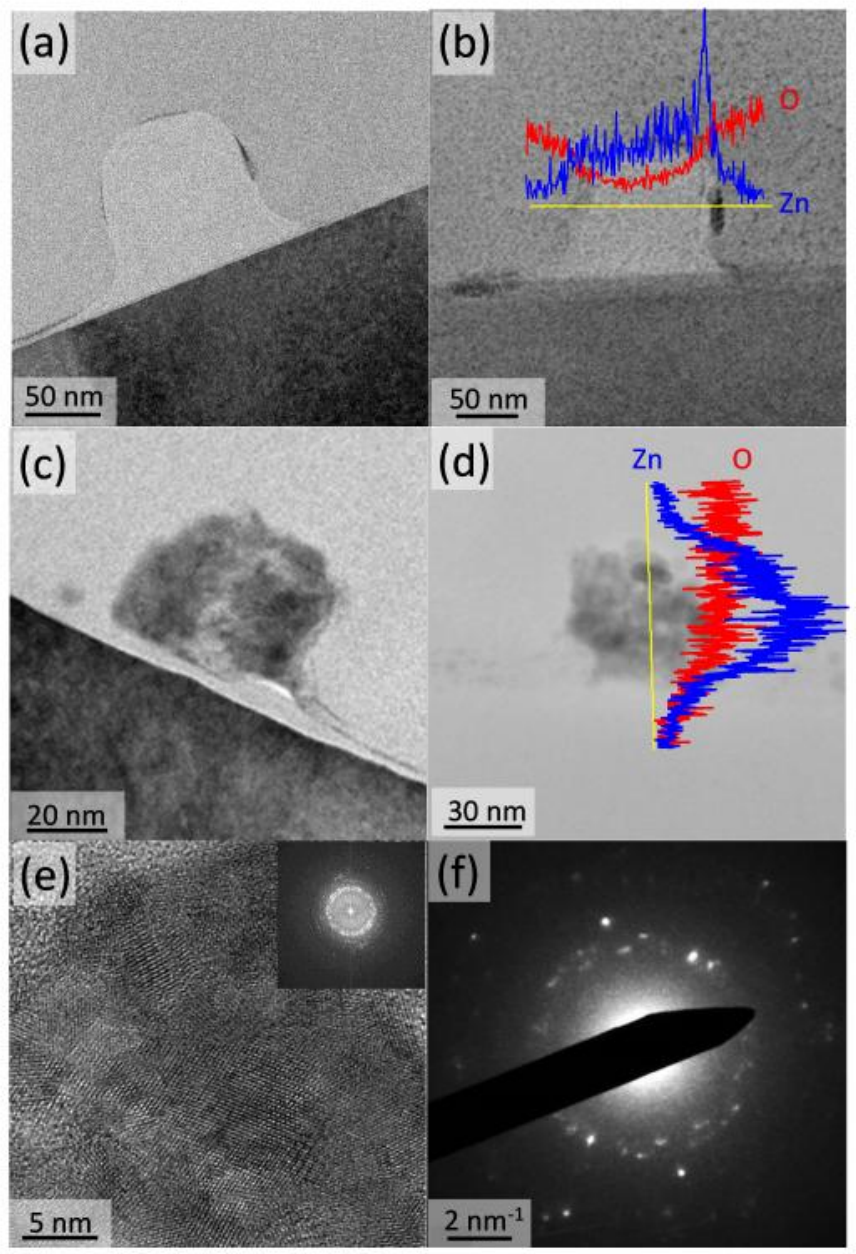

FIG. 3. (a) Cross-sectional TEM micrograph of a SU-8 nanowire template after four infiltration synthesis cycles of $\mathrm{ZnO}$, before removal of the SU-8 matrix. (b) Corresponding bright-field scanning TEM image with superimposed line-scan (yellow line) and EDXS count profiles (red: Oxygen $\mathrm{K}_{\alpha 1}$, and blue: $\mathrm{Zn} \mathrm{K}_{\alpha 1}$ ). Cross-sectional TEM micrographs of an infiltration-synthesized $\mathrm{ZnO}$ nanowire: (c) Low-magnification bright-field image; (d) bright-field scanning TEM image with superimposed line-scan (yellow line) with EDXS count profiles (red: Oxygen $\mathrm{K}_{\alpha 1}$, and blue: $\mathrm{Zn} \mathrm{K}_{\alpha 1}$; (e) high-resolution, bright field image with corresponding FFT (inset); (f) SAED pattern obtained from the center region of $\mathrm{ZnO}$ nanowire cross-section in (e), with closely-spaced, firstand second order pseudo diffraction rings in the middle corresponding to cubic ZnO (JCPDS no. 21-1486, lattice spacing $d=3.39 \AA$ (inner ring) and $3.11 \AA$ (outer ring)). 

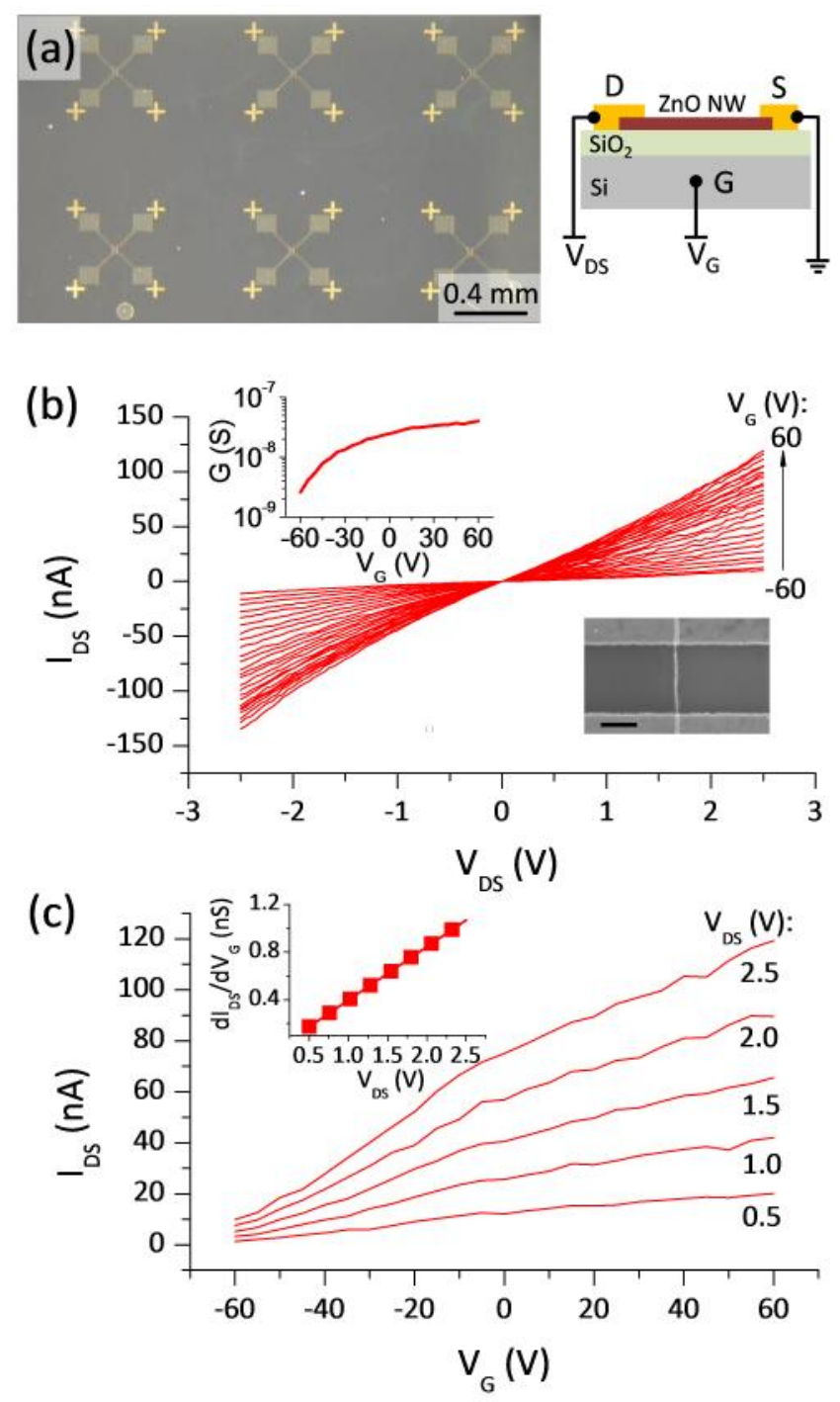

FIG. 4. (a) Top-down optical micrograph of $\mathrm{ZnO}$ nanowire FET arrays fabricated via the infiltration synthesis of $\mathrm{ZnO}$ in patterned SU-8 polymer templates with the schematic (right) depicting the nanowire FET cross-section (S, D, G, and NW denote source, drain, gate, and nanowire). (b) $V_{G^{-}}$-dependent $I_{D S^{-}} V_{D S}$ characteristics of a $\mathrm{ZnO}$ nanowire FET, with the top-left inset showing the dependence of the zero-bias conductance $\left(G\right.$ at $\left.V_{D S}=0\right)$ on $V_{G}$. The bottomright inset shows a SEM micrograph of the nanowire FET channel, with the scale bar marking $500 \mathrm{~nm}$. (c) $V_{D S^{-}}$dependent $I_{D S^{-}} V_{G}$ characteristics of the $\mathrm{ZnO}$ nanowire FET, with the inset showing $V_{D S}$-dependent transconductance $\left(\mathrm{d} I_{D S} / \mathrm{d} V_{G}\right)$. 